\title{
Prescission neutron multiplicity and fission probability from Langevin dynamics of nuclear fission
}

\author{
Gargi Chaudhuri * and Santanu Pal ${ }^{\dagger}$ \\ Variable Energy Cyclotron Centre, 1/AF Bidhan Nagar, Kolkata 700 064, India
}

\begin{abstract}
A theoretical model of one-body nuclear friction which was developed earlier, namely the chaosweighted wall formula, is applied to a dynamical description of compound nuclear decay in the framework of the Langevin equation coupled with statistical evaporation of light particles and photons. We have used both the usual wall formula friction and its chaos-weighted version in the Langevin equation to calculate the fission probability and prescission neutron multiplicity for the compound nuclei ${ }^{178} \mathrm{~W},{ }^{188} \mathrm{Pt},{ }^{200} \mathrm{~Pb},{ }^{213} \mathrm{Fr},{ }^{224} \mathrm{Th}$, and ${ }^{251} \mathrm{Es}$. We have also obtained the contributions of the presaddle and postsaddle neutrons to the total prescission multiplicity. A detailed analysis of our results leads us to conclude that the chaos-weighted wall formula friction can adequately describe the fission dynamics in the presaddle region. This friction, however, turns out to be too weak to describe the postsaddle dynamics properly. This points to the need for a suitable explanation for the enhanced neutron emission in the postsaddle stage of nuclear fission.
\end{abstract}

\section{INTRODUCTION}

The emission of light particles and photons during the prescission stage of a fissioning nucleus has proved to be a useful source of information regarding the dynamics of nuclear fission [1]. In particular, the multiplicity of prescission neutrons, measured over a wide range of excitation energies for a number of compound nuclei, has confirmed [2] that the fission lifetime of a hot nucleus is substantially longer than that determined from the statistical model of Bohr and Wheeler [3]. It is, therefore, natural to expect that a dissipative dynamical model would provide an appropriate description of nuclear fission at high excitation energies. This has given rise to a renewed interest in the works of Kramers [ $\mid$ w who considered the dynamics of nuclear fission to be similar to that of a Brownian particle floating in a viscous heat bath. Though Fokker-Planck equation was initially used to describe such dissipative fission dynamics [5],6], the application of the Langevin equation was found to be more convenient in the later works [1.7].

The Langevin equation has been used extensively in the recent years [1, [11] in order to explain the prescission neutron multiplicity and fission probability of highly excited (typically a few tens of $\mathrm{MeV}$ and above) compound nuclei formed in heavy-ion induced fusion reactions. In these calculations, evaporation of the neutrons and photons (and other light particles) is considered at each instant of time evolution of the fission degrees of freedom. One of the most important inputs to such Langevin dynamical calculations is the dissipative property of the nucleus since it accounts for both the dissipative and the random forces acting on the fission degrees of freedom. While the other inputs to the Langevin equation such as the potential and inertia can be obtained from standard nuclear models, the strength of the dissipative force is still not an unambiguously defined quantity and is often fixed empirically in order to fit the experimental data. In this paper, we shall be mainly concerned with the choice of a dissipative force, based on physical arguments, which can be used in a dynamical description of nuclear fission.

Fröbrich et al. [10] made a detailed study of the fission dynamics and prescission particle emission using the Langevin equation. A comparison of the calculated fission probability and prescission neutron multiplicity excitation functions for a number of nuclei with the experimental data led to a phenomenological shape-dependent nuclear friction in this work. The phenomenological friction turned out to be considerably smaller $(\sim 10 \%)$ than the standard wall formula value for nuclear friction for near-spherical shapes of the compound nucleus whereas a strong increase of this friction was found to be necessary at large deformations. Similar observations were also reported by other workers [11] who obtained a better agreement with the experimental values of prescission neutron multiplicity by reducing the strength of the wall friction. Earlier, Nix and Sierk [12,13] also suggested in their analysis of mean fragment kinetic energy

*Electronic address: gargi@veccal.ernet.in

${ }^{\dagger}$ Electronic address: santanu@veccal.ernet.in 
data that the dissipation is about 4 times weaker than that predicted by the wall-and-window formula of one-body dissipation.

The wall formula for nuclear dissipation was invented long ago in a simple classical picture by extending the mean field concepts to the domain of dissipative dynamics [14]. One crucial assumption of the wall formula concerns the randomization of the particle (nucleon) motion due to the successive collisions it suffers at the nuclear surface. The derivation of the wall formula assumes that the particle motion is fully randomized. It was early realized that any deviation from this full randomization assumption would give rise to a reduction in the strength of the wall formula friction [14]15]. However, it is only recently that a modification of the wall formula has been proposed in which the full randomization assumption is relaxed in order to make it applicable to systems with partly chaotic single-particle motion 16]. In what follows, we shall use the term "chaos- weighted wall formula" (CWWF) for this modified friction in order to distinguish it from the original wall formula (WF) friction. As was shown in Ref. [16], the CWWF friction coefficient $\eta_{c w w f}$ will be given as

$$
\eta_{c w w f}=\mu \eta_{w f},
$$

where $\eta_{w f}$ is the friction coefficient as was given by the original wall formula [14] and $\mu$ is a measure of chaos (chaoticity) in the single-particle motion and depends on the instantaneous shape of the nucleus. The value of chaoticity $\mu$ changes from 0 to 1 as the nucleus evolves from a spherical shape to a highly deformed one. The CWWF friction is thus much smaller than the WF friction for compact nuclear shapes while they become closer at large deformations. Thus the suppression of the strength of wall formula friction achieved in the chaos-weighted wall formula suggests that a lack of full randomization or chaos in single-particle motion can provide a physical explanation for the reduction in strength of friction for compact nuclear shapes as required in the phenomenological friction of Ref. [10].

The main motivation of the present work is to verify to what extent the chaos-weighted wall formula can account for the experimental prescission neutron multiplicity and fission probability data. To this end, we shall use both the CWWF and WF frictions as input to the Langevin equation. The Langevin equation will be solved by coupling it with neutron and $\gamma$ evaporation at each step of its time evolution. Following the work of Fröbrich et al. [10], we shall use a combined dynamical and statistical model for our calculation in which a switching over to a statistical model description will be made when the fission process reaches the stationary regime. The prescission neutron multiplicity and fission probability will be obtained by sampling over a large number of Langevin trajectories. We shall perform calculations at a number of excitation energies for each of the compound nuclei ${ }^{178} \mathrm{~W},{ }^{188} \mathrm{Pt},{ }^{200} \mathrm{~Pb},{ }^{213} \mathrm{Fr},{ }^{224} \mathrm{Th}$, and

${ }^{251}$ Es. A detailed comparison of the calculated values with the experimental data will be presented.

It is worthwhile here to point out a special feature of the present work. We do not have any adjustable parameter in our entire calculation. All the input parameters except the friction coefficients are fixed by standard nuclear models. The chaos weighted wall friction coefficient is obtained following a specific procedure [16] which explicitly considers particle dynamics in phase space in order to calculate the chaoticity factor $\mu$ in Eq.1.1. There is no free parameter in this calculation of friction. In fact, our main aim in this paper is to calculate observable quantities using the theoretically predicted friction and compare them with experimental values in order to draw conclusions regarding the validity of the theoretical model of nuclear friction. As it would turn out, our calculation would not only confirm the theoretical model of the chaos weighted wall friction, it would also provide physical justification for the empirical values of friction used in other works [10]. The present work is thus expected to contribute significantly to our understanding of the dissipative mechanism in nuclear fission.

The paper is organized as follows. The dynamical model along with the necessary input as used in the present calculation will be given in the next section. The details of the calculation will also be given here. The calculated prescission neutron multiplicities and fission probabilities will be compared with the experimental values in Sec.III. A summary of the results along with the conclusions will be presented in the last section.

\section{DETAILS OF THE MODEL}

\section{A. Collective coordinates, potential and inertia}

We have discussed the Langevin equation along with the various input as used in the present calculation in a recent publication [17. We shall use the same definitions and notations in the present work, a brief description of which are as follows. The shape parameters $c, h$ and $\alpha$ as suggested by Brack et al. [18] will be taken as the collective coordinates for the fission degree of freedom. However, we will simplify the calculation by considering only symmetric fission $(\alpha=0)$. We shall further assume in the present work that fission would proceed along the valley of the potential landscape in $(c, h)$ coordinates though we shall consider the Langevin equation in elongation $(c)$ coordinate alone in order to simplify the computation. Consequently, the one-dimensional potential in the Langevin equation 
will be defined as $V(c)=V(c, h)$ at valley. Other quantities such as inertia and friction will also be similarly defined. Since our main concern in the present work is to distinguish between CWWF and WF frictions which give rise to fission rates differing by more than $100 \%$, and it has been already noted that the fission rates in two-dimensional and one-dimensional cases differ by not more than 15\% [19], our approximation of considering fission dynamics in one dimension can be considered adequate for our purpose. Moreover, we have also checked that the prescission neutron multiplicity and fission probability change by less than $5 \%$ when the input fission rates are changed by $15 \%$. Therefore, we estimate that the uncertainty associated with our calculation is rather small allowing us to compare our results with the experimental data.

We shall, therefore, proceed by considering $c$ and its conjugate momentum $p$ as the dynamical variables for fission for our present study and the coupled Langevin equations in one dimension will be given $[\mathbb{8}]$ as

$$
\begin{aligned}
& \frac{d p}{d t}=-\frac{p^{2}}{2} \frac{\partial}{\partial c}\left(\frac{1}{m}\right)-\frac{\partial F}{\partial c}-\eta \dot{c}+R(t), \\
& \frac{d c}{d t}=\frac{p}{m} .
\end{aligned}
$$

The shape-dependent collective inertia and the friction coefficient in the above equations are denoted by $m$ and $\eta$ respectively. The free energy of the system is denoted by $F$ while $R(t)$ represents the random part of the interaction between the fission degree of freedom and the rest of the nuclear degrees of freedom considered collectively as a thermal bath in the present picture. The collective inertia, $m$, will be obtained by assuming an incompressible irrotational flow and making the Werner-Wheeler approximation [20]. The driving force in a thermodynamic system should be derived from its free energy for which we will use the following expression [10] considering the nucleus as a noninteracting Fermi gas:

$$
F(c, T)=V(c)-a(c) T^{2},
$$

where $T$ is the temperature of the system and $a(c)$ is the coordinate-dependent level density parameter which will be chosen following Ref. [10].

The surface of a nucleus of mass number $A$ with elongation and neck coordinates, $c$ and $h$, is defined as

$$
\rho^{2}(z)=\left(1-\frac{z^{2}}{c_{0}^{2}}\right)\left(a_{0} c_{0}^{2}+b_{0} z^{2}\right),
$$

where

$$
\begin{aligned}
c_{0} & =c R, \\
R & =1.16 A^{\frac{1}{3}}
\end{aligned}
$$

and

$$
\begin{aligned}
& a_{0}=\frac{1}{c^{3}}-\frac{b_{0}}{5}, \\
& b_{0}=2 h+\frac{c-1}{2},
\end{aligned}
$$

in cylindrical coordinates. The potential energy $V(c)$ is obtained from the finite-range liquid drop model 21] where we calculate the generalized nuclear energy by double folding the uniform density within the above surface with a Yukawa-plus-exponential potential. The Coulomb energy is obtained by double folding another Yukawa function with the density distribution. The various input parameters are taken from Ref. [21] where they were determined from fitting fission barriers of a wide range of nuclei. The centrifugal part of the potential is calculated using the rigid body moment of inertia. The potential is calculated over a grid of $(c, h)$ values and the valley of the minimum potential located. Potential values along this valley is used in solving the Langevin equation.

The instantaneous random force $R(t)$ is modeled after that of a typical Brownian motion and is assumed to have a stochastic nature with a Gaussian distribution whose average is zero [7]. It is further assumed that $R(t)$ has an extremely short correlation time implying that the intrinsic nuclear dynamics is Markovian. Consequently the strength of the random force can be obtained from the fluctuation-dissipation theorem and the properties of $R(t)$ can be written as

$$
\begin{aligned}
\langle R(t)\rangle & =0, \\
\left\langle R(t) R\left(t^{\prime}\right)\right\rangle & =2 \eta T \delta\left(t-t^{\prime}\right) .
\end{aligned}
$$




\section{B. Dissipation}

One-body dissipation is usually considered to be more successful in describing fission dynamics than two-body viscosity [7,8]. We shall, therefore, use the one-body wall-and-window dissipation $[14$ in the Langevin equation. For the one-body wall dissipation, we shall use the chaos-weighted wall formula (Eq.1.1) introduced in the preceding section. The chaoticity $\mu$ in Eq.1.1 is a measure of chaos in the single-particle motion of the nucleons within the nuclear volume and in the present classical picture, this will be given as the average fraction of the nucleon trajectories that are chaotic when the sampling is done uniformly over the nuclear surface. A trajectory is identified either as a regular or as a chaotic one by considering the magnitude of its Lyapunov exponent and the nature of its variation with time. The details of this procedure are given in Ref. [22]. The chaoticity is calculated for all possible shapes up to the scission configuration. A plot of the variation of chaoticity with elongation can be found in Ref. [17].

In the wall-and-window model of one-body dissipation, the window friction is expected to be effective after a neck is formed in the nuclear system [23]. Further, the radius of the neck connecting the two future fragments should be sufficiently narrow in order to enable a particle that has crossed the window from one side to the other to remain within the other fragment for a sufficiently long time. This is necessary to allow the particle to undergo a sufficient number of collisions within the other side and make the energy transfer irreversible. It therefore appears that the window friction should be very nominal when neck formation just begins. Its strength should increase as the neck becomes narrower reaching its classical value when the neck radius becomes much smaller than the typical radii of the fragments. We however know very little regarding the detailed nature of such a transition. We shall therefore refrain from making any further assumption regarding the onset of window friction. Instead, we shall define a transition point in the elongation coordinate $c_{w i n}$ beyond which the window friction will be switched on. We shall also assume that the compound nucleus evolves into a binary system beyond $c_{w i n}$ and accordingly correction terms for the motions of the centers of mass of the two halves will be applied to the wall formula for $c>c_{\text {win }} 23$.

The choice of a suitable value for the transition point requires some consideartion. We first note that while the window friction makes a positive contribution to the total wall-and-window friction for $c>c_{w i n}$, the center of mass motion correction reduces the wall friction. Therefore, these two contributions cancel each other to a certain extent. Consequently, the resulting wall-and-window friction is not very sensitive to the choice of the transition point. We shall further explore this point quantitatively as follows. When a nucleus moves along the fission path, a neck formation just begins at $c=1.5$. Thus the transition point can lie anywhere beyond this point upto the scission configuratrion. We have performed a few calculations for prescission neutron multiplicity and fission probability with values of $c_{w i n}$ beyond 1.5 the calculated values are in agreement within $5 \%$. Therefore, the value of $c_{w i n}$ is not very critical for our purpose. We shall choose a value for $c_{\text {win }}$ at which the nucleus has a binary shape and the neck radius is half of the radius of either of the would-be fragments. This value of $c_{w i n}$ is thus half-way between its lower and the upper limit in terms of the neck radius. Though such a consideration to choose a value of $c_{w i n}$ is still arbitrary, we have just demonstrated that it will have little influence on our results.

We shall use the following expressions to calculate the wall-and-window friction coefficients $\left(\eta_{w f}\right.$ will henceforth stand for the full wall-and-window friction) [23]:

$$
\eta_{w f}\left(c<c_{w i n}\right)=\eta_{w a l l}\left(c<c_{w i n}\right)
$$

where

$$
\eta_{w a l l}\left(c<c_{w i n}\right)=\frac{1}{2} \pi \rho_{m} \bar{v} \int_{z_{m i n}}^{z_{\max }}\left(\frac{\partial \rho^{2}}{\partial c}\right)^{2}\left[\rho^{2}+\left(\frac{1}{2} \frac{\partial \rho^{2}}{\partial z}\right)^{2}\right]^{-\frac{1}{2}} d z
$$

and

$$
\eta_{w f}\left(c \geq c_{w i n}\right)=\eta_{w a l l}\left(c \geq c_{w i n}\right)+\eta_{w i n}\left(c \geq c_{w i n}\right)
$$

where

$$
\begin{aligned}
\eta_{\text {wall }}\left(c \geq c_{\text {win }}\right)= & \frac{1}{2} \pi \rho_{m} \bar{v}\left\{\int_{z_{\min }}^{z_{N}}\left(\frac{\partial \rho^{2}}{\partial c}+\frac{\partial \rho^{2}}{\partial z} \frac{\partial D_{1}}{\partial c}\right)^{2}\left[\rho^{2}+\left(\frac{1}{2} \frac{\partial \rho^{2}}{\partial z}\right)^{2}\right]^{-\frac{1}{2}} d z\right. \\
& \left.+\int_{z_{N}}^{z_{\max }}\left(\frac{\partial \rho^{2}}{\partial c}+\frac{\partial \rho^{2}}{\partial z} \frac{\partial D_{2}}{\partial c}\right)^{2}\left[\rho^{2}+\left(\frac{1}{2} \frac{\partial \rho^{2}}{\partial z}\right)^{2}\right]^{-\frac{1}{2}} d z\right\}
\end{aligned}
$$


and

$$
\eta_{w i n}\left(c \geq c_{w i n}\right)=\frac{1}{2} \rho_{m} \bar{v}\left(\frac{\partial R}{\partial c}\right)^{2} \Delta \sigma .
$$

In the above equations, $\rho_{m}$ is the mass density of the nucleus, $\bar{v}$ is the average nucleon speed inside the nucleus and $D_{1}, D_{2}$ are the positions of the centers of mass of the two parts of the fissioning system relative to the center of mass of the whole system. $z_{\min }$ and $z_{\max }$ are the two extreme ends of the nuclear shape along the $z$ axis and $z_{N}$ is the position of the neck plane that divides the nucleus into two parts. In the window friction coefficient, $R$ is the distance between centers of mass of future fragments and $\Delta \sigma$ is the area of the window between the two parts of the system.

The wall friction coefficients given by (Eqs.2.6 and 2.8) are obtained [14] under the assumption of a fully chaotic nucleon motion within the nuclear volume. However, a fully chaotic motion is achieved only when the nuclear shape is extremely irregular whereas the nucleon motion is partly chaotic in varying degrees for typical nuclear shapes through which a nucleus evolves when it undergoes fission. We have already argued in the preceding section that for such cases, the chaos weighted wall friction $\left(\eta_{c w w f}\right)$ should be employed instead of the original wall friction. Accordingly, we shall replace Eqs.2.6 and 2.8 by their chaos weighted versions and the chaos-weighted wall-and-window friction (denoted henceforth by $\eta_{c w w f}$ ) is subsequently obtained as

$$
\eta_{c w w f}\left(c<c_{w i n}\right)=\mu(c) \eta_{w a l l}\left(c<c_{w i n}\right),
$$

and

$$
\eta_{c w w f}\left(c \geq c_{w i n}\right)=\mu(c) \eta_{\text {wall }}\left(c \geq c_{\text {win }}\right)+\eta_{\text {win }}\left(c \geq c_{\text {win }}\right) .
$$

Defining a quantity $\beta(c)=\eta(c) / m(c)$ as the reduced friction coefficient, its dependence on the elongation coordinate is shown in Fig. 17 for both the WF and CWWF frictions for the ${ }^{213} \mathrm{Fr}$ nucleus. A strong suppression of the original wall formula friction for compact shapes of the nucleus can be immediately noticed in the CWWF friction. This implies that the chaoticity is very small for near spherical shapes $(c \sim 1)$, the physical picture behind which is as follows. A particle moving in a spherical mean field represents a typical integrable system and its dynamics is completely regular. When the boundary of the mean field is set into motion (as in fission), the energy gained by the particle at one instant as a result of a collision with the moving boundary is eventually fed back to the boundary motion in the course of later collisions. An integrable system thus becomes completely nondissipative in this picture resulting in a vanishing friction coefficient. This aspect has been investigated extensively on earlier occasions [15], 16] and has been found to be valid for any generic integrable system. The reduction in the strength of the wall friction as shown in Fig. 11 thus becomes evident from chaos considerations. The phenomenological reduced friction obtained in Ref. [10] is also shown in this figure. Though the one-body friction with the CWWF agrees qualitatively with the phenomenological friction for $c<1.5$, it is beyond its scope to explain the steep increase of phenomenological friction for $c>1.5$. We shall discuss this point further while presenting the results.

\section{Combined dynamical and statistical model calculation}

In our calculation, we first specify the entrance channel through which a compound nucleus is formed. Assuming complete fusion of the target with the projectile, the spin distribution of the compound nucleus is usually found to follow the analytical form

$$
\frac{d \sigma(l)}{d l}=\frac{\pi}{k^{2}} \frac{(2 l+1)}{1+\exp \frac{\left(l-l_{c}\right)}{\delta l}}
$$

where the parameters $l_{c}$ and $\delta l$ should be obtained by fitting the experimental fusion cross sections. It is however found that these parameters for different systems follow an approximate scaling [i] and we shall, therefore, use the scaled values of these parameters. The initial spin of the compound nucleus will be obtained by sampling the above spin distribution function. The initial distribution of the coordinates and momenta $(c, p)$ is assumed to be close to equilibrium and hence their initial values are chosen from sampling random numbers following the Maxwell-Boltzmann distribution. With these initial conditions, the Langevin equations (Eq.2.1) are numerically integrated following the procedure outlined in Ref. [7]. The total excitation energy $\left(E^{*}\right)$ of the compound nucleus can easily be obtained from the beam energy of the projectile and energy conservation in the form

$$
E^{*}=E_{i n t}+V(c)+p^{2} / 2 m
$$


gives the intrinsic excitation energy $E_{\text {int }}$ and the corresponding nuclear temperature $T=\left(E_{\text {int }} / a\right)^{1 / 2}$ at each time step of integration. The centrifugal potential is included in $V(c)$ in the above equation.

We shall also consider neutron and giant dipole $\gamma$ evaporation at each Langevin time step $\tau$ in the following manner [10]. We shall first calculate the neutron and $\gamma$ decay widths, $\Gamma_{n}$ and $\Gamma_{\gamma}$, by using the inverse cross-section formula as given in Ref. [1]. These widths depend upon the temperature, spin and the mass number of the compound nucleus and hence are to be evaluated at each interval of time evolution of the compound nucleus. We shall next decide whether any evaporation takes place during the interval or not by first calculating the ratio $x=\tau / \tau_{\text {tot }}$ where $\tau_{\text {tot }}=\hbar / \Gamma_{\text {tot }}$ and $\Gamma_{t o t}=\Gamma_{n}+\Gamma_{\gamma}$. We shall then choose a random number $r$ by sampling from a uniformly distributed set between 0 and 1. If we find $r<x$, it will be interpreted as emission of either a neutron or a $\gamma$ during that interval. The type of the emitted particle is next decided by a Monte Carlo selection where it is considered as a neutron if $0 \leq r \leq \Gamma_{n} / \Gamma_{t o t}$, $r$ being again sampled from a uniform distribution of random numbers $(0 \leq r \leq 1)$, and as a $\gamma$ otherwise. This procedure simulates the law of radioactive decay for the emitted particles. The energy of the emitted particle is then obtained by another Monte Carlo sampling of its energy spectrum. The intrinsic excitation energy, mass and spin of the compound nucleus are recalculated after each emission. The spin of the compound nucleus is reduced only in an approximate way by assuming that each neutron or a $\gamma$ carries away $1 \hbar$ angular momentum. A Langevin trajectory will be considered as undergone fission if it reaches the scission point $\left(c_{s c i}\right)$ in course of its time evolution. Alternately it will be counted as an evaporation residue event if the intrinsic excitation energy becomes smaller than either the fission barrier or the binding energy of a neutron. The calculation proceeds until the compound nucleus undergoes fission or ends up as an evaporation residue. The number of emitted neutrons and photons is recorded for each fission event. This calculation is repeated for a large number of Langevin trajectories and the average number of neutrons emitted in the fission events will give the required prescission neutron multiplicity. The fission probability will be obtained as the fraction of the trajectories which have undergone fission.

The above scheme can however take an extremely long computer time particularly for those compound nuclei whose fission probability is small. We shall therefore follow a combined dynamical and statistical model, first proposed by Mavlitov et al. [9], in the present calculation. In this model, we shall first follow the time evolution of a compound nucleus according to the Langevin equations as described above for a sufficiently long period during which a steady flow across the fission barrier is established. Beyond this period, a statistical model for compound nucleus decay is expected to be a equally valid and more economical in terms of computation. We shall therefore switch over to a statistical model description after the fission process reaches the stationary regime. We shall, however, require the fission width along with the neuton and $\gamma$ widths in the statistical branch of the calculation. This fission width should be the stationary limit of the fission rate as determined by the Langevin equation. Though analytic solutions for fission rates can be obtained in special cases [4,24] assuming a constant friction, this is not the case with the CWWF friction which is not constant and is strongly shape dependent. Thus it becomes necessary to find a suitable parametric form of the numerically obtained stationary fission widths using the CWWF (and also WF) frictions in order to use them in the statistical branch of our calculation. The details of this procedure is given in Ref. [17] following which we shall calculate all the required fission widths for the present work.

\section{RESULTS}

We have calculated the prescission neutron multiplicity and the fission probability for a number of compound nuclei formed in heavy-ion induced fusion reactions. We have used both the CWWF and WF frictions in our calculation. Figure 2 shows the results for prescission neutron multiplicity along with the experimental data. A number of systematic features can be observed from these results. First, the prescission neutron multiplicity values calculated with the CWWF and WF frictions are very close at smaller excitation energies, though at higher excitation energies, the WF predictions are larger than those obtained with the CWWF. This aspect is present in the decay of all the compound nuclei which we consider here and can be qualitatively understood as follows. The magnitude of the CWWF friction being smaller than that of the WF friction, fission rate with the CWWF friction is higher than that obtained with the WF friction. We have shown earlier 17] that the stationary fission width with the CWWF friction is about twice of that with the WF friction. However at a low excitation energy where a compound nucleus is formed with a low value of spin, the fission barrier is high and both the CWWF and WF fission widths turn out to be many times smaller than the neutron width. The neutrons, therefore, have enough time to be emitted long before a compound nucleus undergoes fission irrespective of its dynamics being controlled by either the CWWF or the WF frictions. Thus the prescission neutron multiplicities are rather insensitive to fission time scales at lower excitation energies. On the other hand, a compound nucleus is formed with a larger spin at higher excitation energies resulting in a reduction of the fission barrier. The fission time scales and the neutron lifetimes start becoming comparable at higher excitation energies and less neutrons are predicted from calculations with the CWWF than those with the WF. The prescission 
neutron multiplicity thus becomes capable of discriminating between different models of nuclear friction at higher excitation energies of the compound nucleus.

A similar explanation also holds for the systematic variation of the calculated prescission neutron multiplicities with respect to the mass number of the compound nucleus. We find that the WF prediction for prescission neutrons starts getting distinct from that of the CWWF at smaller values of the excitation energy with increasing mass number of the compound nucleus. Since the fission barrier decreases with the increasing mass of a compound nucleus, the fission time scales and the neutron lifetimes become comparable for heavier compound nuclei at lower excitation energies. This results in a fewer neutrons from calculations with the CWWF than those with the WF as one considers heavier compound nuclei.

A number of interesting points can be noted while comparing the calculated values with the experimental data. For the compound nucleus ${ }^{178} \mathrm{~W}$, the available experimental points [25] are at low excitation energies and therefore, cannot distinguish between the calculated values using the CWWF and WF frictions, which are almost identical. The calculated values slightly overestimate the prescission neutron multiplicity compared to the experimental data. A more extensive set of experimental values for prescission neutron multiplicity are available for the compound nuclei ${ }^{188} \mathrm{Pt},{ }^{200} \mathrm{~Pb},{ }^{213} \mathrm{~F}$, r and ${ }^{224} \mathrm{Th} 25$ 27 covering a wider range of excitation energy in which the calculated values with the CWWF and WF differ. Clearly, the CWWF predicted values give excellent agreement with the experimental data for these compound nuclei whereas the WF predictions are considerably higher. However, similar conclusions cannot be drawn for the heavier nucleus ${ }^{251}$ Es. It appears that the WF predictions are closer to the experimental data [28,26,25] whereas the CWWF predictions are somewhat lower. We shall return to this point later for a detailed discussion. For the present, we shall consider the results of fission probability calculations.

The calculated and experimental values of fission probability are shown in Fig. 3 for four compound nuclei. Experimental data for ${ }^{224} \mathrm{Th}$ is rather scanty and fission probability for ${ }^{251} \mathrm{Es}$ is almost $100 \%$. Hence they are excluded from the present discussion. The calculated values of fission probability complements the picture of fission dynamics which was obtained while discussing the prescission neutron data. The fission probability is found to be more sensitive to the choice of friction at lower excitation energies than at higher excitations. The CWWF predicted fission probabilities are larger than those from the WF predictions. Moreover, the CWWF predictions are consistently closer to the experimental values of fission probability than those from the WF predictions.

In order to gain further insight into the dynamics of fission, we have also calculated the presaddle and postsaddle (saddle to scission) contributions to the multiplicity of prescission neutrons. Figure 1 shows the results obtained with both the CWWF and WF frictions. For all the cases, starting from almost zero multiplicity at small excitation energies, the postsaddle contribution increases at higher excitation energies. It is further observed that the postsaddle neutron multiplicities calculated with the CWWF and WF frictions are almost same for all the compound nuclei over the range of excitation energies considered here. This would be due to the fact that the number of postsaddle neutrons depends on the time scale of descent from the saddle to the scission. This, in turn, will depend upon the strength of the friction between the saddle and the scission and we have already seen in Fig. 1 that the CWWF and WF frictions are indeed close at large deformations. We shall next compare the presaddle contributions calculated with the CWWF and WF frictions for each of the nuclei under consideration. We immediately notice that the WF predictions are consistently larger than those from the CWWF at higher excitation energies. This gives rise to the enhancement of the WF prediction for total prescission multiplicity compared to that from the CWWF prediction, which we have already noticed in Fig. 2 and have discussed earlier. Since the CWWF predicted neutron multiplicities agree with the experimental values for the nuclei ${ }^{178} \mathrm{~W},{ }^{188} \mathrm{Pt},{ }^{200} \mathrm{~Pb},{ }^{213} \mathrm{Fr}$, and ${ }^{224} \mathrm{Th}$, we conclude that the chaos-weighted wall formula provides the right kind of friction to describe the presaddle dynamics of nuclear fission.

While comparing the relative importance of the presaddle and postsaddle neutrons, we further note that the postsaddle neutrons are more frequently emitted from heavier compound nuclei. For ${ }^{251}$ Es, most of the prescission neutrons predicted by the CWWF are accounted for by the postsaddle neutrons. The underlying physical picture can be described as follows. When a compound nucleus is formed in a heavy-ion induced fusion reaction, its spin distribution is assumed to be given by Eq.2.12. If the compound nucleus is formed with a spin at which there is no fission barrier, its transition to the scission point will be essentially considered as postsaddle dynamics. In order to simplify our discussion, let us assume that most of the compound nuclei at a given excitation energy are formed with the spin $l_{0}$ of Eq2.12 and let $l_{b}$ be the limiting spin value at which the fission barrier vanishes. We can then find a critical excitation energy, $E_{c r i t}$, above which $l_{0}$ becomes greater than $l_{b}$ and most of the fission dynamics at excitations above this critical value can be considered as comprising of only postsaddle trajectories. In Fig. 5, we have plotted the fraction of neutrons emitted in the postsaddle stage as a function of the excitation energy for a number of compound nuclei. The critical excitation energy for each nucleus is also given in this plot. We have used the CWWF predicted neutron multiplicities for this plot where we find that the critical excitation energy decreases with increase in the compound nuclear mass. Thus the dominance of postsaddle neutrons sets in at lower excitation energies for heavier nuclei which, in turn, gives rise to the increase in the fraction of postsaddle neutrons with increasing mass of the compound nucleus. 
Though the above discussion clearly establishes the importance of postsaddle neutrons for a very heavy compound nucleus, the number of postsaddle neutrons calculated with the CWWF friction still falls short of making the total prescission multiplicity equal to the experimental values for ${ }^{251}$ Es. We consider the apparent better agreement between the WF predicted prescission neutron multiplicity and the experimental data for ${ }^{251}$ Es as shown in Fig. 2 2 as a mere coincidence and we do not find any physical justification for abandoning the chaos-weighted factor in one-body friction for such heavy nuclei. Instead, we feel that the mechanism of neutron emission in the postsaddle stage requires a closer scrutiny essentially because the nucleus becomes strongly deformed beyond the saddle point. The neutron decay width of such a strongly deformed nucleus could be quite different from that of the equilibrated near-spherical nucleus which we use in our calculation. In particular, the neutron-to-proton ratio is expected to be higher in the neck region than that in the nuclear bulk and this can cause more neutrons to be emitted. Further, dynamical effects such as inclusion of the neck degree of freedom in the Langevin equation can influence the time scale of the postsaddle dynamics and hence the number of emitted neutrons. Such possibilities should be examined in future for a better understanding of the postsaddle dynamics of nuclear fission.

\section{SUMMARY AND CONCLUSIONS}

We have applied a theoretical model of one-body nuclear friction, namely the chaos-weighted wall formula, to a dynamical description of compound nuclear decay where fission is governed by the Langevin equation coupled with the statistical evaporation of light particles and photons. We have used both the normal wall formula and its modified form with the chaos-weighted factor in our calculation in order to find its effect on the fission probabilities and prescission neutron multiplicities for a number of compound nuclei. The strength of the chaos-weighted wall formula friction being much smaller than that of the wall formula, the fission probabilities calculated with the CWWF are found to be larger than those predicted with the WF friction. On the other hand, the prescission neutron multiplicities predicted with the CWWF friction turn out to be smaller than those using the WF friction. Both the prescission neutron multiplicity and fission probability calculated with the CWWF friction for the compound nuclei ${ }^{178} \mathrm{~W},{ }^{188} \mathrm{Pt},{ }^{200} \mathrm{~Pb}$, ${ }^{213} \mathrm{Fr}$, and ${ }^{224} \mathrm{Th}$ agree much better with the experimental data compared to the predictions of the WF friction.

We have subsequently investigated the role of presaddle and postsaddle neutrons at different excitation energies for different compound nuclei. It has been shown that the majority of the prescission neutrons are emitted in the postsaddle stage for a very heavy nucleus like ${ }^{251}$ Es. The CWWF friction, however, cannot produce enough neutrons to match the experimental prescission multiplicities for such a nucleus. It is, therefore, possible that in the postsaddle region, either the fission dynamics gets considerably slowed down or the neutrons are more easily emitted. These aspects require further studies before we draw conclusions regarding the postsaddle dynamics of nuclear fission.

The presaddle neutrons are however found to account for most of the prescission neutrons for lighter nuclei at lower excitation energies. On the basis of the comparison of the calculated prescission multiplicities with experimental data as given in the preceding section, we can conclude that the chaos-weighted wall formula friction can adequately describe the fission dynamics in the presaddle region.

\section{ACKNOWLEDGMENTS}

The authors are grateful to Nicolas Carjan for making valuable suggestions during the course of the work.

[1] P. Fröbrich and I.I. Gontchar, Phys. Rep. 292, 131 (1998).

[2] M. Thoennessen and G.F. Bertsch, Phys. Rev. Lett. 71, 4303 (1993).

[3] N. Bohr and J.A. Wheeler, Phys. Rev. 56, 426 (1939).

[4] H.A. Kramers, Physica (Amsterdam) 4, 284 (1940).

[5] P. Grange and H.A. Weidenmüller, Phys. Lett. 96B, 26 (1980).

[6] P. Grange, Q. Li-Jang, and H.A. Weidenmüller, Phys. Rev. C 27, 2063 (1983).

[7] Y. Abe, S. Ayik, P.-G. Reinhard, and E. Suraud, Phys. Rep. 275, 49 (1996).

[8] T. Wada, Y. Abe, and N. Carjan, Phys. Rev. Lett. 70, 3538 (1993).

[9] N.D. Mavlitov, P. Fröbrich, and I.I. Gonchar, Z. Phys. A 342, 195 (1992)

[10] P. Fröbrich, I.I. Gontchar, and N.D. Mavlitov, Nucl. Phys. A556, 281 (1993). 
[11] K. Pomorski, B. Nerlo-Pomorska, A. Surowiec, M. Kowal, J. Bartel, K. Dietrich, J. Richert, C. Schmitt, B. Benoit, E. de Goes Brennand, L. Donadille, and C. Badimon, Nucl. Phys. A679, 25 (2000).

[12] J.R. Nix and A.J. Sierk in Proceedings of the International School-Seminar on Heavy Ion Physics, Dubna, USSR, 1986, Report No. JINR-D7-87-68 (1987), p.453.

[13] J.R. Nix and A.J. Sierk in Proceedings of the 6th Adriatic Conference on Nuclear Physics: frontiers of Heavy Ion Physics, Dubrovnik, Yogoslavia, 1990, edited by N. Cindro et al. (World Scientific, Singapore, 1990), p. 333.

[14] J. Blocki, Y. Boneh, J.R. Nix, J. Randrup, M. Robel, A.J. Sierk, and W.J. Swiatecki, Ann. Phys. (N.Y.) 113, 330 (1978).

[15] S.E. Koonin and J. Randrup, Nucl. Phys. A289, 475 (1977).

[16] S. Pal and T. Mukhopadhyay, Phys. Rev. C54, 1333 (1996).

[17] Gargi Chaudhuri and S. Pal, Phys. Rev. C63, 064603 (2001).

[18] M. Brack, J. Damgard, A.S. Jensen, H.C. Pauli, V.M. Strutinsky, and C.Y. Wong, Rev. Mod. Phys. 44, 320 (1972).

[19] T. Wada, N. Carjan, and Y. Abe, Nucl. Phys. A538, 283c (1992).

[20] K.T.R. Davies, A.J. Sierk, and J.R. Nix, Phys. Rev. C13, 2385 (1976).

[21] A.J. Sierk, Phys. Rev. C33, 2039 (1986).

[22] J. Blocki, F. Brut, T. Srokowski, and W.J. Swiatecki, Nucl. Phys. A545, 511c (1992).

[23] A.J. Sierk and J. R. Nix, Phys. Rev. C21, 982 (1980).

[24] I.I. Gontchar, P. Fröbrich, and N.I. Pischasov, Phys. Rev. C47, 2228 (1993).

[25] J.O. Newton, D.J. Hinde, R.J. Charity, R.J. Leigh, J.J.M. Bokhorst, A. Chatterjee, G.S. Foote, and S. Ogaza, Nucl. Phys. A483, 126 (1988).

[26] D.J. Hinde, D. Hilscher, H. Rossner, B. Gebaure, M. Lehmann, and M. Wilpert, Phys. Rev. C45, 1229 (1992).

[27] D.J. Hinde, H. Ogata, M. Tanaba, T. Shimoda, N. Takahashi, A. Shinohara, S. Wakamatsu, K. Katori, and H. Okamura, Phys. Rev. C39, 2268 (1989).

[28] H. Rossner, D.J. Hinde, J.R. Leigh, J.P. Lestone, J.O. Newton, J.X. Wei, and S. Elfstrom, Phys. Rev. C45, 719 (1992).

[29] R.J. Charity, J.R. Leigh, J.J.M. Bokhorst, A. Chatterjee, G.S. Foote, D.J. Hinde, J.O. Newton, S. Ogaza, and D. Ward, Nucl. Phys. A457, 441 (1986).

[30] J.S. Forster, L.V. Mitchell, J.U. Andersen, A.S. Jensen, E. Laegsgard, W.M. Gibson, and K. Reichelt, Nucl. Phys. A464, 497 (1987).

[31] D.J. Hinde, R.J. Charity, G.S. Foote, J.R. Leigh, J.O. Newton, S. Ogaza, and A. Chatterjee, Nucl. Phys. A452, 550 (1986). 


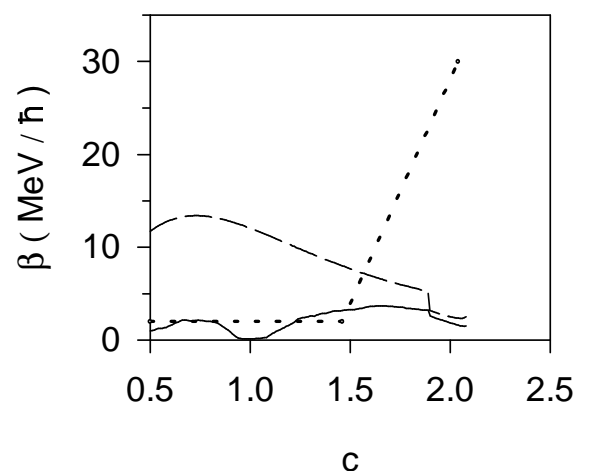

FIG. 1. Reduced one-body friction coefficient $\beta$ with chaos-weighted wall formula (solid line) and wall formula (dashed line) frictions for ${ }^{213} \mathrm{Fr}$. The phenomenological reduced coefficient (dotted line) from Ref. [10] is also shown. 


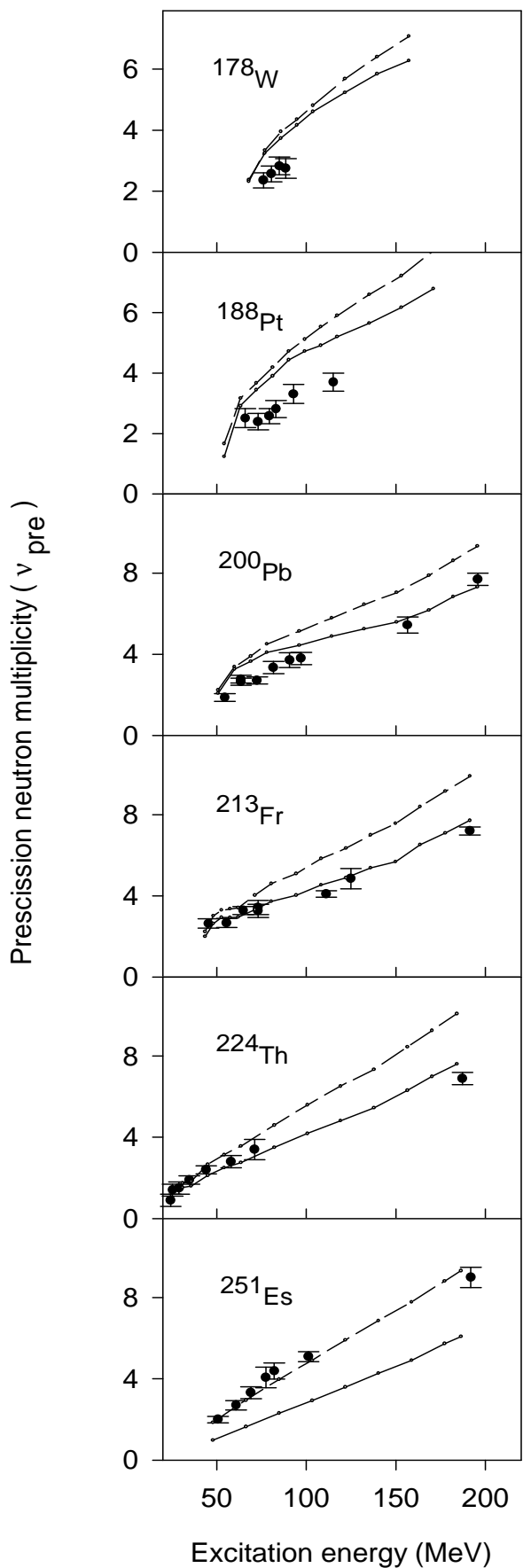

FIG. 2. Prescission neutron multiplicities calculated with the CWWF friction are shown as points connected by solid lines whereas those calculated with the WF friction are shown as points connected by dashed lines. The experimental data for ${ }^{178} \mathrm{~W}$, ${ }^{188} \mathrm{Pt},{ }^{200} \mathrm{~Pb},{ }^{213} \mathrm{Fr},{ }^{224} \mathrm{Th}$, and ${ }^{251} \mathrm{Es}$ are from Refs. [25], [25,26], [25-27], [25-27], [26,28], and [25,26], respectively. 

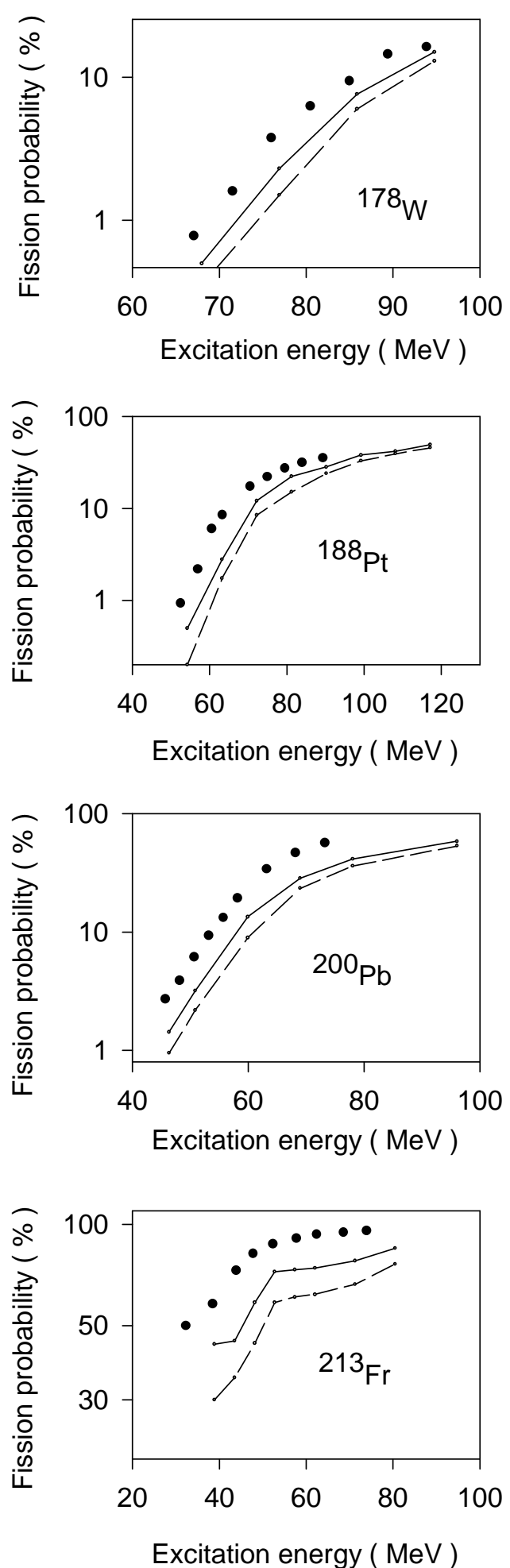

FIG. 3. Fission probabilities calculated the CWWF friction are shown as points connected by solid lines whereas those calculated with the WF friction are shown as points connected by dashed lines. The experimental data for ${ }^{178} \mathrm{~W},{ }^{188} \mathrm{Pt},{ }^{200} \mathrm{~Pb}$, and ${ }^{213} \mathrm{Fr}$ are from Refs. [29], [29], [30], and [31], respectively. 


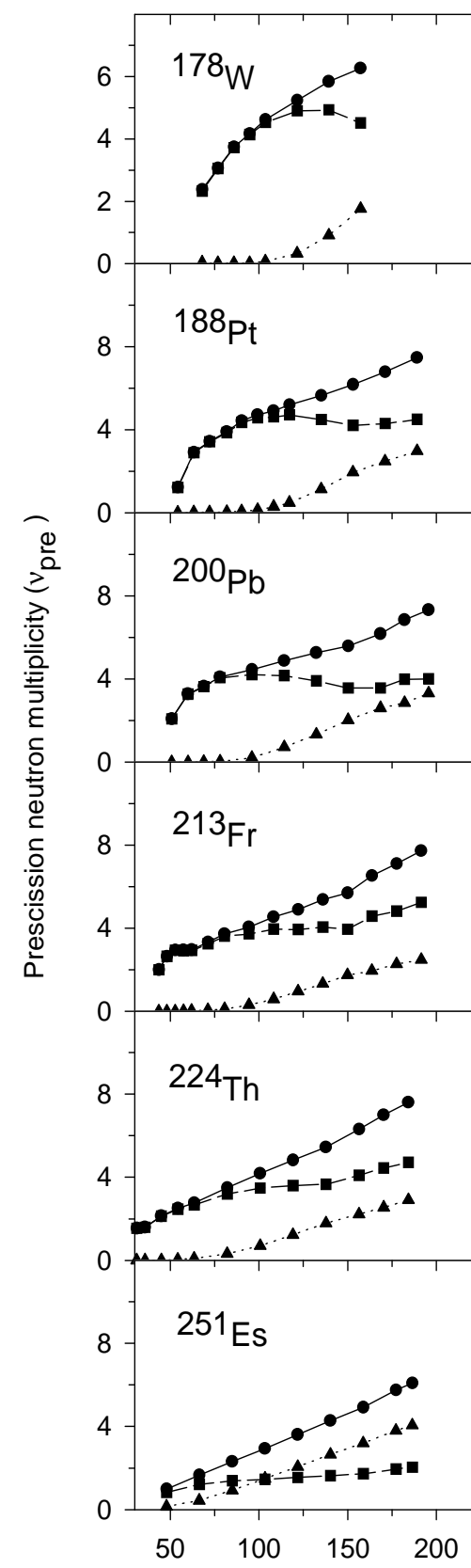

Excitation energy (MeV)

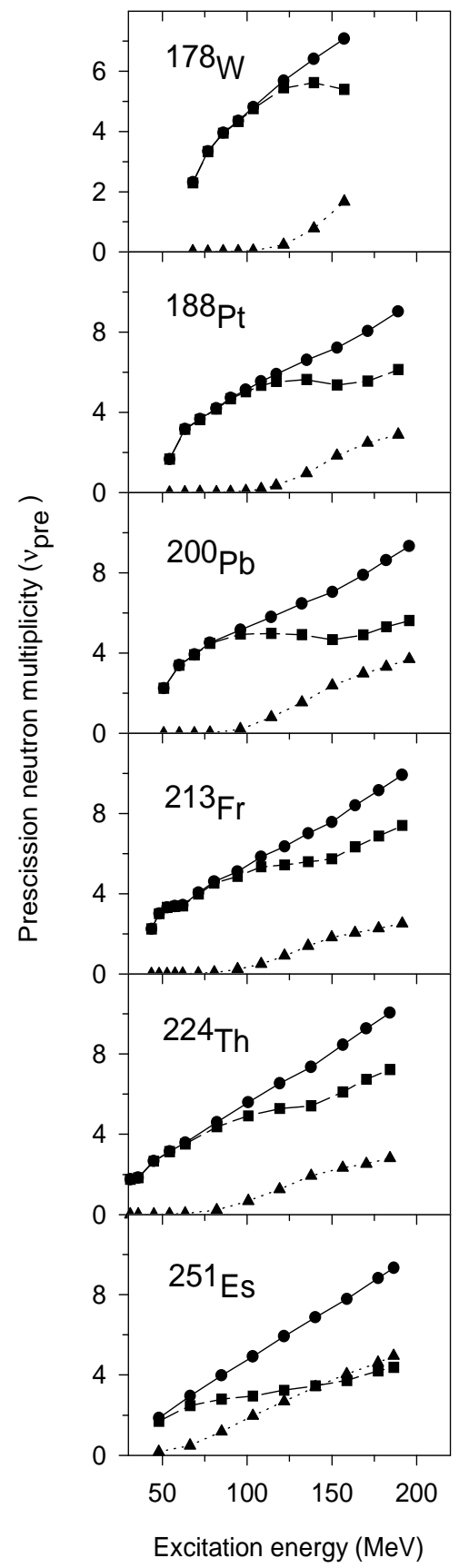

FIG. 4. Neutrons emitted during the presaddle and postsaddle (saddle to scission) stages of fission. Figures in the left panel show values calculated with the CWWF friction whereas those in right panel are obtained with the WF friction. In each plot, the solid circles, the solid squares and the solid triangles represent the total number of prescission neutrons, the number of presaddle neutrons and the number of postsaddle neutrons, respectively. 


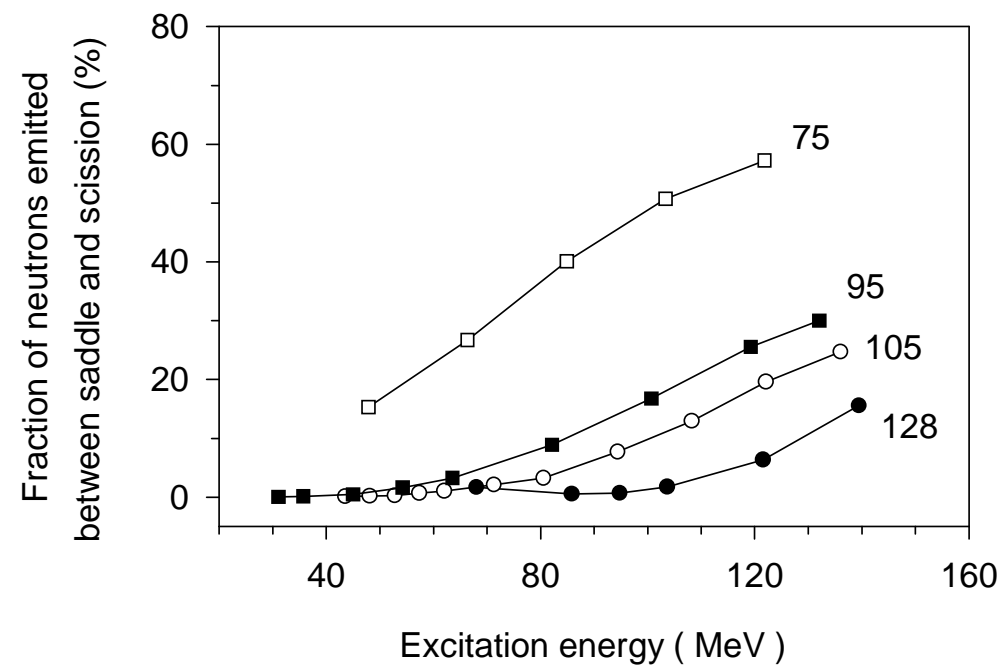

FIG. 5. Fraction of neutrons emitted between saddle and scission is shown as a function of excitation energy for different compound nuclei. The the open square, the solid square, the open circle and the solid circle represent the calculated values for ${ }^{251} \mathrm{Es},{ }^{224} \mathrm{Th},{ }^{213} \mathrm{Fr}$, and ${ }^{178} \mathrm{~W}$, respectively. The critical excitation energy (in units of MeV), as defined in the text, is indicated for each nucleus. 